\title{
The Effects of Operating Parameters on Micro-Droplet Formation in a Piezoelectric Inkjet Printhead Using a Double Pulse Voltage Pattern
}

\author{
Huey-Jiuan Lin ${ }^{1}$, Hsuan-Chung $\mathrm{Wu}^{2}$, Tzu-Ray Shan ${ }^{2}$ and Weng-Sing Hwang ${ }^{2}$ \\ ${ }^{1}$ Department of Materials Science and Engineering, National United University, MiaoLi, Taiwan, R. O. China \\ ${ }^{2}$ Department of Materials Science and Engineering, National Cheng Kung University, Tainan, Taiwan, R. O. China
}

\begin{abstract}
The formation and ejection behavior of droplets created by a squeeze mode piezoelectric inkjet printing device using a double pulse voltage pattern are investigated in this study. Two types of fluid, de-ionized (DI) water and ethylene glycol, are used. The effects of operating frequency, positive voltage keeping time and pulse voltage magnitude on the volume and velocity of the droplets are discussed. The experimental results are consistent with the propagation theory of acoustic waves. The maximum allowable pulse frequencies in DI water and ethylene glycol are 2,000 and $22,700 \mathrm{~Hz}$ respectively. If the positive voltage keeping time equals the time required for the acoustic waves to propagate through the printhead, optimal ejection behavior is achieved. As the pulse voltage increases, both the velocity and volume of each droplet become larger.
\end{abstract}

(Received September 23, 2005; Accepted December 5, 2005; Published February 15, 2006)

Keywords: inkjet printing, squeeze-tube type, micro-droplet, acoustic wave theory

\section{Introduction}

The development of manufacturing technologies has been driven by the need for automation, miniaturization, and the reduction of costs and environmental impact. To meet these needs, inkjet printing technology is an increasingly attractive alternative for the distribution and patterning of material in a wide variety of applications. Other than its application in computer printers, the prospect of adopting inkjet printing technology in various high-tech processes is very promising.

Solder-Jet technology ${ }^{1)}$ has been applied in ball grid array (BGA) and flip-chip electronics packaging processes. More than 1,440 tin/lead (63/37) solder balls, each approximately $70 \mu \mathrm{m}$ in diameter, were successfully deposited onto a test substrate at $220^{\circ} \mathrm{C}$. Compared with conventional soldering processes, Solder Jet-based processes not only reduce the number of processing steps but also are environmentally friendly, numeric processor driven, and highly flexible. Hence, they can increase productivity and lower costs.

Inkjet printing technology also has great potential in flat panel display applications. ${ }^{2)}$ Organic light-emitting diodes (OLEDs) are one of the most promising flat panel technologies for the future. However, due to the limited selection of materials, they are usually fabricated by thin film deposition and evaporation process with subsequent patterning through lithography. The processes, along with the required masks, are very complicated and expensive. Tremendous benefits can be obtained if arrays of organic light emitting materials can be deposited directly by inkjet printing methods. The current practice for polymer light emitting diodes (PLEDs) is deposition through spin coating. However, this is restricted to the fabrication of a single color. With the application of inkjet printing technology, it is possible to deposit tiny pixels of red, green, and blue elements at the same time to produce the color filters. Compared to the spin coating process, inkjet printing technology has the benefits of high resolution, low cost, simpler processing, a high yield of materials utilization and the capability to produce large panels. Inkjet printing technology also provides a unique process for the fabrication of micro-lens arrays as well as complex three-dimensional structures. ${ }^{3)}$

A variety of actuation methods, including piezoelectric, thermal bubble, electro-static and acoustic ${ }^{4)}$ etc., have been adopted to eject droplets. Among these methods, the actuations using piezoelectric and thermal bubble devices are the most mature and popular systems for commercial inkjet printers. For thermal bubble devices, the ejection of liquid droplets from nozzle is induced by the formation of a vapor bubble in the ink through the heating of a resistive film. For the piezoelectric inkjet printing, the liquid droplets are ejected from the nozzle by the displacement of a piezoelectric diaphragm that is coupled to the fluid. Thus, this method can be used for the ejection and dispensing of polymers and liquid metals. The adoption of the piezoelectric inkjet printing technology in the manufacturing of the electronic industry is then very much desired.

As a framework for the experimental study of droplet ejection in inkjet printing, Bugdayci et al. ${ }^{5)}$ used the theories of stress and energy conservation to calculate the relationship between the voltage applied to the piezoelectric transducer (PZT) and the induced pressure for a squeeze type piezoelectric printhead. Bogy and Talke ${ }^{6)}$ present experimental observations and concluded that the piezoelectric inkjet phenomena were related to the propagation and reflection of acoustic waves within the inkjet cavity. Expansions and contractions of the tube resulting from an applied rectangular voltage pulse will generate a negative and positive pressure wave, which, after appropriate reflections, reinforce or interferes with each other to enhance or impede the velocity of the ejected drops. The optimum pulse width was equal to ration of cavity length and speed on sound in liquid. Shield ${ }^{7)}$ used both experimentation and two-dimensional numerical simulation to study the ejection behavior of droplets of DI water and ethylene glycol. The simulation results were compared with the experimental results. However, the effects of the experimental conditions were insufficiently discussed. Chen $^{8)}$ investigated pressure generation in the ink flow channel and ink droplet formation for a piezoelectric print- 


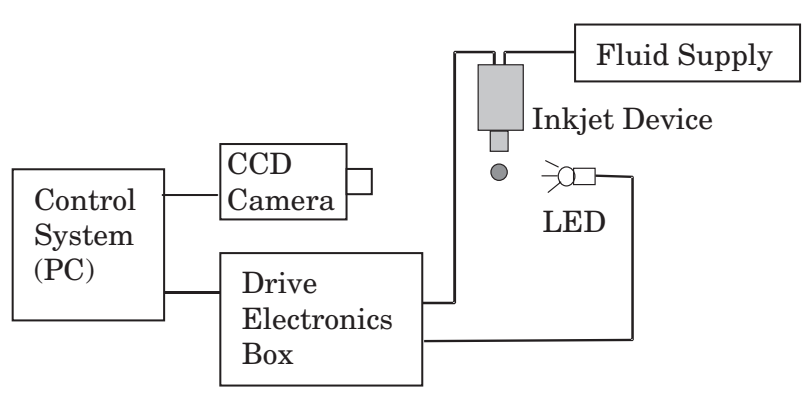

Fig. 1 A schematic diagram of the experimental setup.

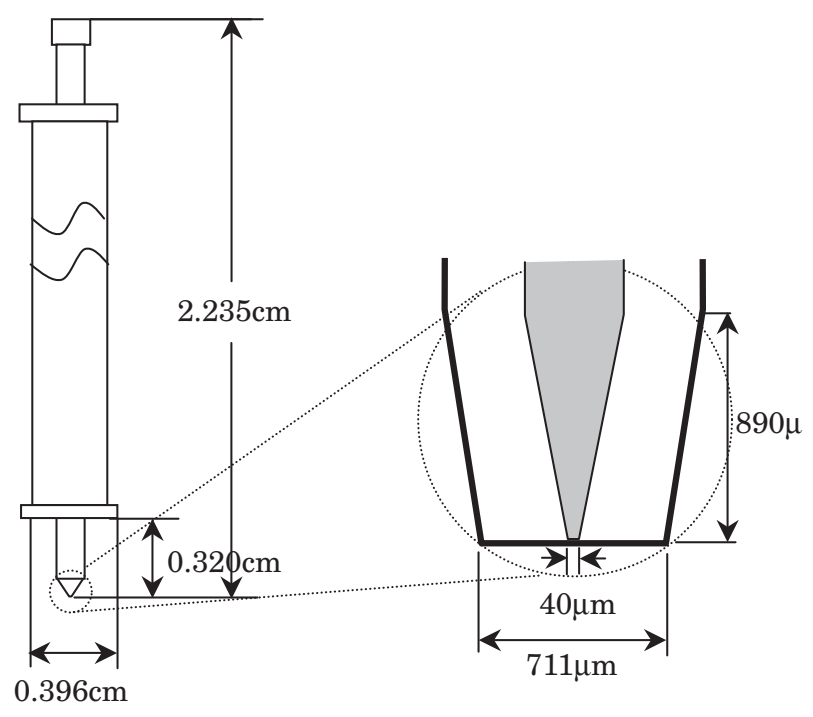

Fig. 2 Geometry and dimensions of the inkjet printhead device.

head. ANSYS and the characteristic method were used to solve the one-dimensional wave equation to obtain the transient pressure and velocity variations in the flow channel of the printhead.

The printing quality and resolution of an inkjet printhead are known to be closely related to the characteristics of the ejected droplet. The flow behavior of droplet depends strongly on the physical design parameters, fluid of the droplet. In this paper, we investigate the effects of droplet ejection behavior (including droplet volume and velocity) under different operating conditions and fluid types and discuss in terms of acoustic wave theory.

\section{Experimental}

\subsection{Observation of droplet ejection process}

An experimental system, as shown schematically in Fig. 1, is set up to observe the droplet ejection process at different time instants and to measure the volume of the droplet. The jetting device consists of a piezoelectric printhead which is surrounded by metal for protection, and connects to a fluid supply chamber as well as the drive electronic box. The dimensions of the jetting device are shown in Fig. 2. The drive electronics box includes a strobe driver and pulse controller. The strobe driver controls the delay time of the LED. The printhead is driven by input the pulse voltage,
Table 1 Physical property of DI water and ethylene glycol.

\begin{tabular}{lcc}
\hline & DI water & Ethylene glycol \\
\hline Surface tension $(\mathrm{N} / \mathrm{m})$ & 0.072 & 0.047 \\
Viscosity $(\mathrm{N} /(\mathrm{s} . \mathrm{m}))$ & 0.89 & 1.12 \\
Density $\left(\mathrm{Kg} / \mathrm{m}^{3}\right)$ & 1000.0 & 1120.0 \\
Acoustic velocity $(\mathrm{m} / \mathrm{s})$ & 1435 & 1658 \\
\hline
\end{tabular}

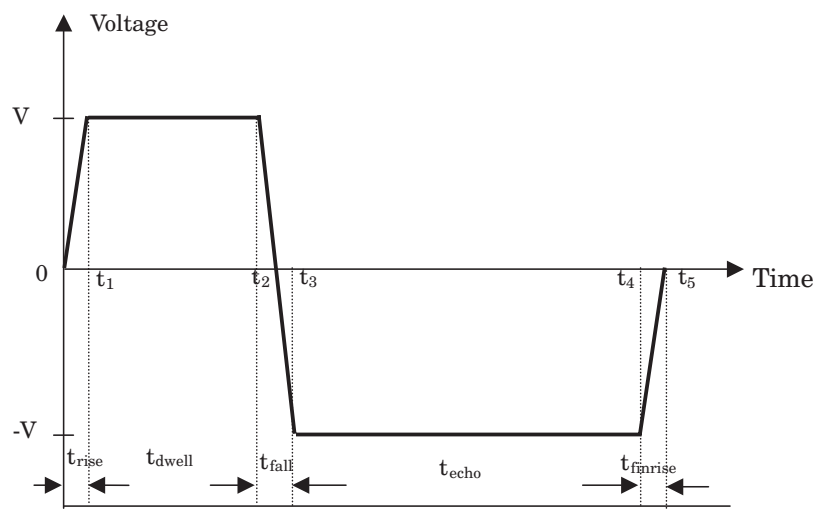

Fig. 3 Double pulse voltage pattern employed in this study.

pulse width and frequency from pulse controller. All of the data could be input from personal computer. The ejected droplet images are captured by the CCD camera, then digitized by the frame grabber, stored as a file in the computer and shown on the monitor.

Two different liquids, DI water and ethylene glycol, are employed, because of their material properties and because they are commonly used as the base fluids for inks in inkjet printing. DI water has higher surface tension and lower viscosity than. Ethylene glycol is. The surface tension coefficients, densities, viscosities, and acoustic velocities of these liquids are shown in Table 1.

\subsection{Setup of experimental conditions}

At the beginning of the experiment, the voltage pattern and LED delay time are input. The double pulse pattern, as shown in Fig. 3, is chosen in this study. All five time intervals, $t_{\text {rise }}$, $t_{\text {dwell }}, t_{\text {fall }}, t_{\text {echo }}, t_{\text {finrise }}$ and the voltage amplitude $(V)$ as well as pulse frequency can be individually varied. The pulse frequency is defined as the number of voltage pulses per second. $t_{\text {rise }}\left(=t_{1}\right)$ is the time required for the voltage to rise from zero to $V$. The polarization causes the piezoelectric transducer (PZT) tube to move outward in the radial direction, which results in a negative pressure distribution in the liquid inside cavity. The positive voltage $V$ is maintained for the duration $t_{\text {dwell }}\left(=t_{2}-t_{1}\right)$. The PZT has no displacement during this time. However, the pressure is propagated continuously in the cavity. $t_{\text {fall }}\left(=t_{3}-t_{2}\right)$ is the time required for the voltage to drop from $V$ to $-V$. The depolarization causes the PZT to move radically inward and results in an positive pressure profile. The negative voltage $-V$ is maintained for the duration $t_{\text {echo }}\left(=t_{4}-t_{3}\right)$. The PZT has no displacement during this time. $t_{\text {finrise }}\left(=t_{5}-t_{4}\right)$ is the time required for the voltage to rise from $-V$ to zero. The return of the PZT to the neutral state results in a second 
Table 2 Parameters used for the study of frequency effects.

\begin{tabular}{|c|c|c|}
\hline Liquid & $\begin{array}{c}\text { Experimental Conditions } \\
t_{\text {rise }-} t_{\text {dwell }} t_{\text {fall }}-t_{\text {echo- }} t_{\text {finrise }} V \_F r\end{array}$ & $\begin{array}{l}\text { Control variables } \\
\text { Frequency }(\mathrm{Hz})\end{array}$ \\
\hline DI water & 1.5_15_3_30_1.5_8_Fr & $\begin{array}{c}500,1000,1500,2000,2500 \\
3000,3500,4000\end{array}$ \\
\hline $\begin{array}{l}\text { Ethylene } \\
\text { glycol }\end{array}$ & 1_13_2_26_1_15_Fr & $\begin{array}{c}2000,3000,4000,8000,12000, \\
16000,20000,22700\end{array}$ \\
\hline
\end{tabular}

Table 3 Parameters used for the study of pulse time effects.

\begin{tabular}{|c|c|c|}
\hline Liquid & $\begin{array}{c}\text { Experimental Conditions } \\
t_{\text {rise }-} t_{\text {dwell }-} t_{\text {fall }} t_{\text {echo- }} t_{\text {finrise_}} V \_F r\end{array}$ & $\begin{array}{c}\text { Control variables } \\
\text { Pulse Duration- } t_{\text {dwell }}(\mu \mathrm{s})\end{array}$ \\
\hline DI water & $1 \_t_{\text {dwell_}} 2 \_2 t_{\text {dwell- }} 1 \_10 \_1500$ & $8,10,12,14,16,18,20,22$ \\
\hline $\begin{array}{l}\text { Ethylene } \\
\text { glycol }\end{array}$ & $1 \_t_{\text {dwell} \_2 \_} 2 t_{\text {dwell_-1_15_1500 }}$ & $10,11,12,13,14,15,16,17$ \\
\hline
\end{tabular}

Table 4 Parameters used for the study of voltage effects.

\begin{tabular}{|c|c|c|}
\hline Liquid & $\begin{array}{c}\text { Experimental Conditions } \\
t_{\text {rise }-} t_{\text {dwell }-} t_{\text {fall }}-t_{\text {echo }}-t_{\text {finrise }-} V \_F r\end{array}$ & $\begin{array}{c}\text { Control variables } \\
\text { Voltage }(V)\end{array}$ \\
\hline DI water & 1_15_2_30_1_V_2000 & $8,9,10$ \\
\hline $\begin{array}{l}\text { Ethylene } \\
\text { glycol }\end{array}$ & 1_13_2_26_1_V_2000 & $14,17,20$ \\
\hline
\end{tabular}

pressure drop in the liquid contained in the cavity. After $t_{5}$, the PZT exerts no influence on the liquid in the cavity. A sufficient time of $t_{\text {rise }}, t_{\text {fall }}$ and $t_{\text {finrise }}$ is necessary to output the full desired voltage and thus the PZT will reach the desired expansion. Therefore, the voltage pulse output by the pulse controller to the printhead must meet with the requirements given below.

$$
\frac{V}{t_{\text {rise }}} \leq 15 \quad \frac{V}{t_{\text {fall }}} \leq 15 \quad \frac{V}{t_{\text {finrise }}} \leq 15
$$

That is, the slope of voltage variation can not be more than $15 \mathrm{vol} / \mu \mathrm{s}$. At meanwhile, better droplet ejection is obtained when $t_{\text {echo }}=2 t_{\text {dwell }}$. And, the voltage amplitude must be high enough to cause droplet ejection, but not so high that the ejection becomes chaotic and difficult to observe.

In order to keep the number of experimental parameters at a minimum in this study, we have used only pulse frequency $(F r)$, pulse time $\left(t_{\text {dwell }}\right)$ and voltage amplitude $(V)$ as the parameters and discuss their influence on the droplet ejection behavior. For conveniently, the notation $\left[t_{\text {rise }-} t_{\text {dwell }}-t_{\text {fall }}-t_{\text {echo }}-t_{\text {finrise }}-V F r\right]$ is used to represent the experimental conditions of voltage rise time ( $\mu$ s)_positive voltage keeping time $(\mu s)$ _voltage fall time $(\mu s)$ negative voltage keeping time $(\mu s)$ _voltage final rise time $(\mu s)$ _voltage $(V)$ operating frequency $(\mathrm{Hz})$. A summary of the conditions for the experiments in this study is given in Tables 2, 3, and 4.

\subsection{Measurement of droplet volume and flight distance}

Figure 4 illustrates how the droplet diameter and flight distance are determined from the experimental observations. The width of the glass tube underneath nozzle $(A)$ is $711 \mu \mathrm{m}$. The flight distance of the droplet can be calculated according

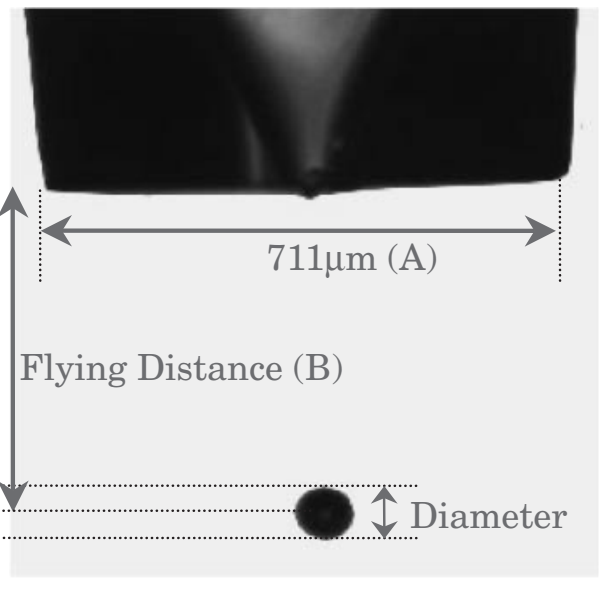

Fig. 4 Measurements of diameter and flight distance of the liquid droplet from an experimental observation.

to the relationship $(B / A) \times 711 \mu \mathrm{m}$. In order to measure the droplet volume, an image in which the droplet is spherical is selected. The diameter can be calculated using the equation $d=(C / A) \times 711 \mu \mathrm{m}$, and the liquid droplet volume can be calculated using $V=\left(4 \pi r^{3}\right) / 3$, with $r$ being the radius of the liquid droplet. The droplet velocity is determined by measuring the flight distances $B$ at time $t$ and $B^{\prime}$ at time $t^{\prime}$, and is calculated by the following equation.

$$
v=\frac{B-B^{\prime}}{t-t^{\prime}}
$$

\section{Results and Discussion}

\subsection{Basics for piezoelectric inkjet}

A squeeze mode piezoelectric printhead employed in this study consists of a cylindrical piezoelectric tube that surrounds a glass capillary insert, as shown schematically in Fig. 5. The capillary insert and the PZT tube are of equal length and are bonded together. The tube assembly is supported on both ends by flexible washers and is coaxially aligned with channels of equal diameter. The two channels and the PZT tube assembly form a cavity of length $L$. The front end of the cavity is terminated with a silicon plate which contains a hole, called nozzle. The back end of the cavity is connected to a supply tube of larger diameter. When a voltage pulse is applied across the PZT tube, a radial displacement of the tube is initiated. Depending on the polarization of the PZT, the inner radius can increase or decrease with a voltage increase, and causes the propagation of a pressure wave inside the cavity. The pressure waves propagate in both directions and will be reflected or transmitted depending on the structure of cavity.

The end of tube with nozzle plate can be considered as approximately closed, because that the nozzle opening is a small fraction of the tube cross-sectional area. However, the supply end can be considered as approximately open, since the inside diameter of the supply tube is larger than that of the squeeze tube. From the analysis of wave propagation theory, a pressure wave will change signs when it reflects from an open end and retain its sign as it reflects from a closed end. 


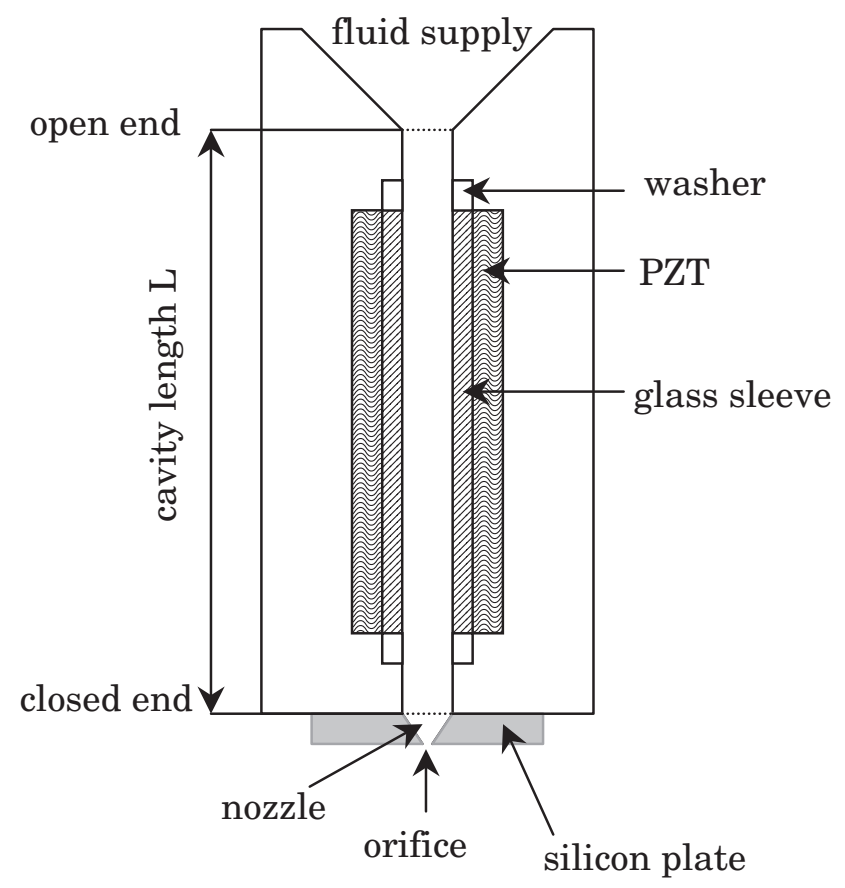

Fig. 5 A schematic diagram of a squeeze mode piezoelectric inkjet printhead.

Bogy ${ }^{6)}$ had proposed an optimum pulse width, $t_{\mathrm{opt}}$, calculating as following

$$
t_{\mathrm{opt}}=t_{2}-t_{1}=\frac{L}{v_{\mathrm{aco}, \mathrm{liq}}}
$$

$v_{\text {aco,liq }}$ is the velocity of acoustic wave propagation in the liquid. If the width of voltage pulse is equal to $t_{\mathrm{opt}}$, the positive pressure wave reflects from nozzle end with maximum amplitude could be expected. If the pressure is high enough, the liquid will be ejected at $t_{0}+1.5 t_{\text {opt }}$. The theoretical value of optimal pulse time for DI water is

$$
t_{\mathrm{opt}, \mathrm{wat}}=\frac{2.235(\mathrm{~cm})}{1435(\mathrm{~m} / \mathrm{s})}=15.57 \mu \mathrm{s}
$$

and ethylene glycol is

$$
t_{\text {opteth }}=\frac{2.235(\mathrm{~cm})}{1658(\mathrm{~m} / \mathrm{s})}=13.48 \mu \mathrm{s}
$$

In this research, we modified the wave propagation theory proposed by Bogy, which the rectangular pulse was applied. The propagation and reflections of pressure wave induced by double pulse voltage pattern in the cavity could be assumed as shown in Fig. 6. There are five stages in a double pulse voltage. The rising time $\left(t_{\text {rise }}\right)$, falling time $\left(t_{\text {fall }}\right)$ and final rising time $\left(t_{\text {finrise }}\right)$ are short and can be neglected here. A negative acoustic wave is represented beneath the horizontal line, and a positive one is represented above the horizontal line. In the first stage, an initial rising voltage from zero to $V$ volts is applied at $t=t_{0}$. A negative pressure is induced by the sudden expansion of the tube at this instant, as shown in Fig. 6(a). In the second stage, the voltage is kept at $V$ volts with a pulse width of $t_{\text {dwell }}$, and the tube is assumed to be without deformation or oscillation. The negative pressure wave will divide in two and the split waves propagate in

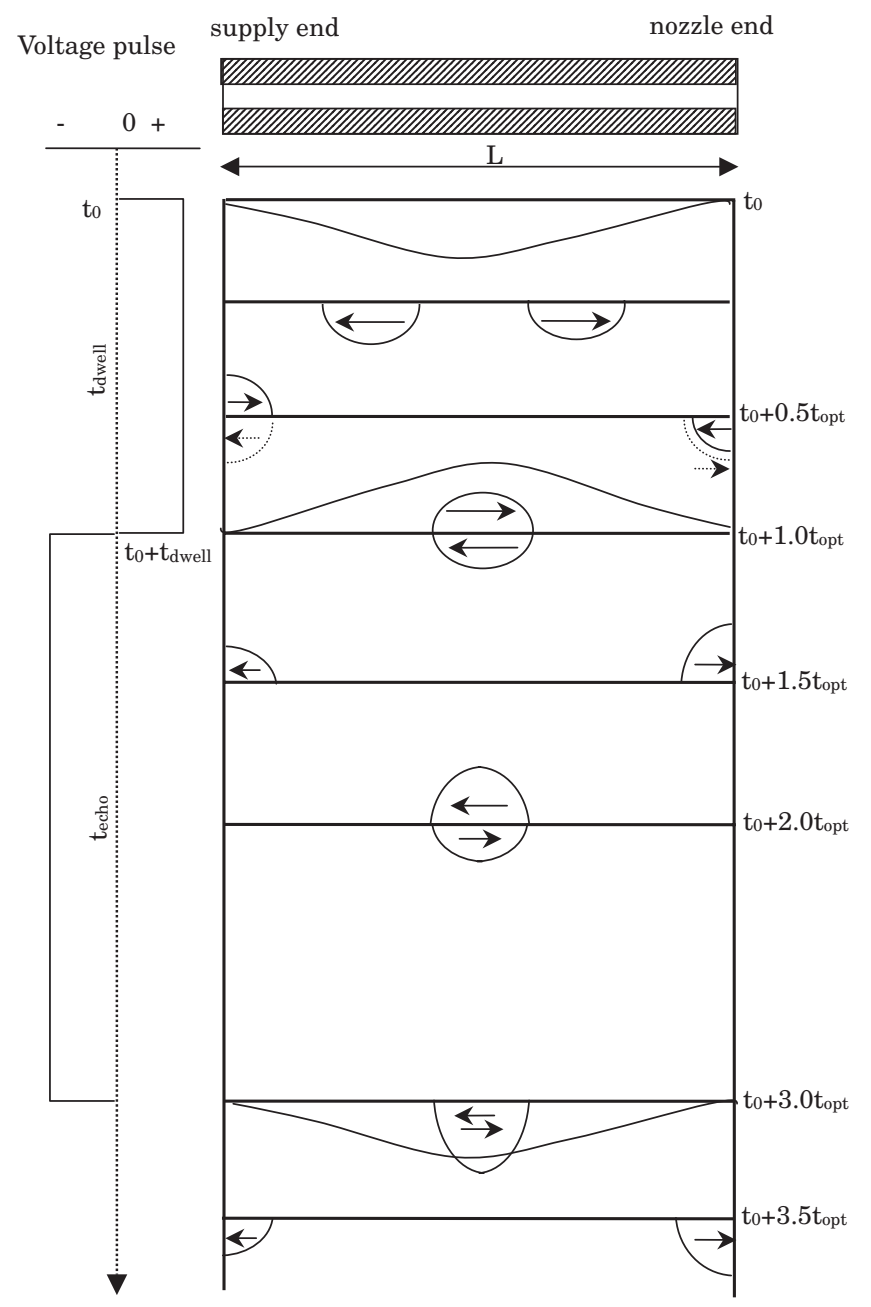

Fig. 6 Schematic illustrations of wave propagation and reflection of pressure waves with double pulse voltage pattern.

opposite directions (toward the open end and the closed end) with the amplitude of half the initial pressure, as shown in Fig. 6(b). If the $t_{\text {dwell }}$ is equal to $t_{\mathrm{opt}}$, the split pressure waves will be reflected from the two ends as shown in Fig. 6(c). The dotted lines represent the pressure waves before reflection and the solid lines represent the pressure waves after reflection. Figure 6(d) shows both the pressure waves after each has traveled a distance $L$. In the third stage, the voltage steps down to negative $V$ volts, the tube should contract and thereby add a new positive pressure in the liquid. The result is that the left propagating negative pressure wave is dampened into a small positive pressure wave and the right propagating pressure wave is amplified, as shown in Fig. 6(e). Therefore, by applying the voltage down-step at exactly $t_{1}+t_{\text {opt }}$, the amplitude of the pressure wave should be optimally enhanced. In the four stage, the voltage is kept at $-V$ volts with a pulse width of $t_{\text {echo }}$. It could be found that at this instant $t_{1}+1.5 t_{\text {opt }}$ [Fig. 6(f)], the pressure at the nozzle reaches its maximum value. This should be the most favorable condition for drop ejection if the amplitude of the pressure wave is high enough.

In the five stage, the voltage is increased from $-V$ volts to zero. This will cause an expansion of the tube and adds a negative pressure at this instant, as shown in Fig. 6(g). This 


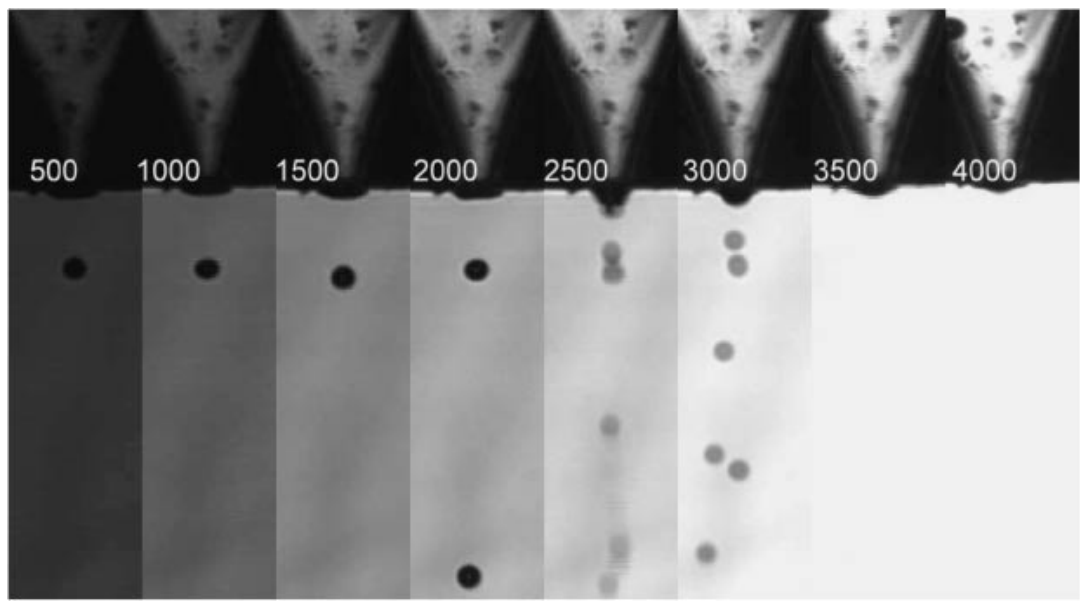

(a) 1.5_15_3_30_1.5_8_Fr with frequency varying from $500 \mathrm{~Hz}$ to $4,000 \mathrm{~Hz}$.

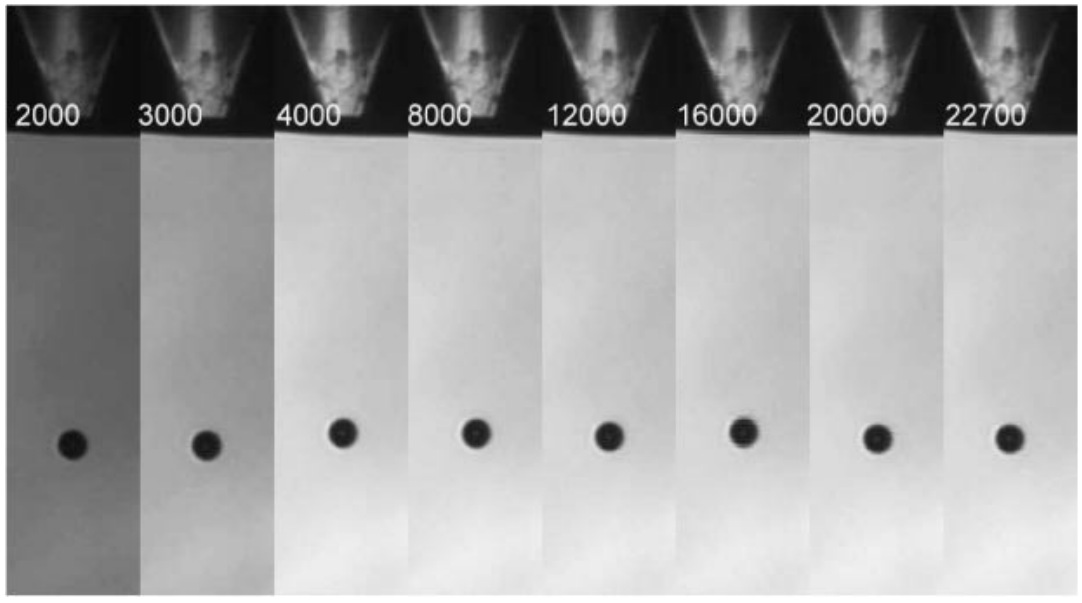

(b) $1 \_13 \_2 \_26 \_1 \_15 \_F r$ with frequency varying from $2,000 \mathrm{~Hz}$ to $22,700 \mathrm{~Hz}$.

Fig. 7 Experimental observations of droplet with various frequencies at $150 \mu$ s for (a) DI water, (b) ethylene glycol.

new negative pressure wave is thereby superimposed on those remaining from the previous stage. If the voltage applied at five stage is just at the instant $t_{0}+3 t_{\text {opt }}$, the maximum negative pressure waves should arrive at the nozzle at instant $t_{0}+3.5 t_{\mathrm{opt}}$. This negative pressure wave will cause the ejected drop to neck and break.

On the basis of this discussion, the setting of the pulse width of $t_{\mathrm{dwell}}$ and $t_{\mathrm{echo}}$ are very important and influences the distribution of pressure waves in the cavity. The optimum pulse width of $t_{\text {dwell }}$ and $t_{\text {echo }}$ is equal to $t_{\mathrm{opt}}$ and $2 t_{\mathrm{opt}}$ respectively.

\subsection{Operating frequency effects}

Table 2 shows the experimental conditions used to discuss the effects of operating frequency on droplet ejection behavior for DI water and ethylene glycol. The operating conditions for DI water inkjet experiment are 1.5_15_3_30_1.5_8_Fr with various frequencies $(F r=500$, $1000,1500,2000,2500,3000,3500$ and $4000 \mathrm{~Hz}) . t=0$ is defined as the time of pulse initiation. The experimental conditions for Ethylene glycol liquid are 1_13_2_26_1_15_Fr, $F r=2000,3000,4000,8000,12000,16000,20000$, and $22700 \mathrm{~Hz}$
Figure 7 shows the images taken at $t=150 \mu$ s for DI water and ethylene glycol droplets ejected at various pulse frequencies. It is found that when the frequency is higher than $2000 \mathrm{~Hz}$, the chaotic droplet ejection phenomena will be induced for DI water, as shown in Fig. 7(a). However, for ethylene glycol only one, spherical droplet is obtained even the pulse frequency higher than $22700 \mathrm{~Hz}$, as shown in Fig. 7(b). The chaotic droplet ejection is induced at high frequency, because that the previous pressure wave in the cavity does not have enough time to decay fully and, interacts with the pressure wave produced by the next voltage pulse. In contrast, lower frequencies will result in a lower droplet ejection rate, causing decreased production efficiency. In order to optimize efficiency, the operating frequency should be as high as possible without causing chaotic droplet ejection. In this study, the optimum frequencies for DI water and ethylene glycol are 2000 and $22700 \mathrm{~Hz}$, respectively.

The decay time of pressure wave is determined by the properties of ink fluids, and could be calculated by the optimal pulse frequency, as shown below.

$$
t_{\text {decay }}=\frac{1}{\text { frequency } y_{\mathrm{opt}}}
$$




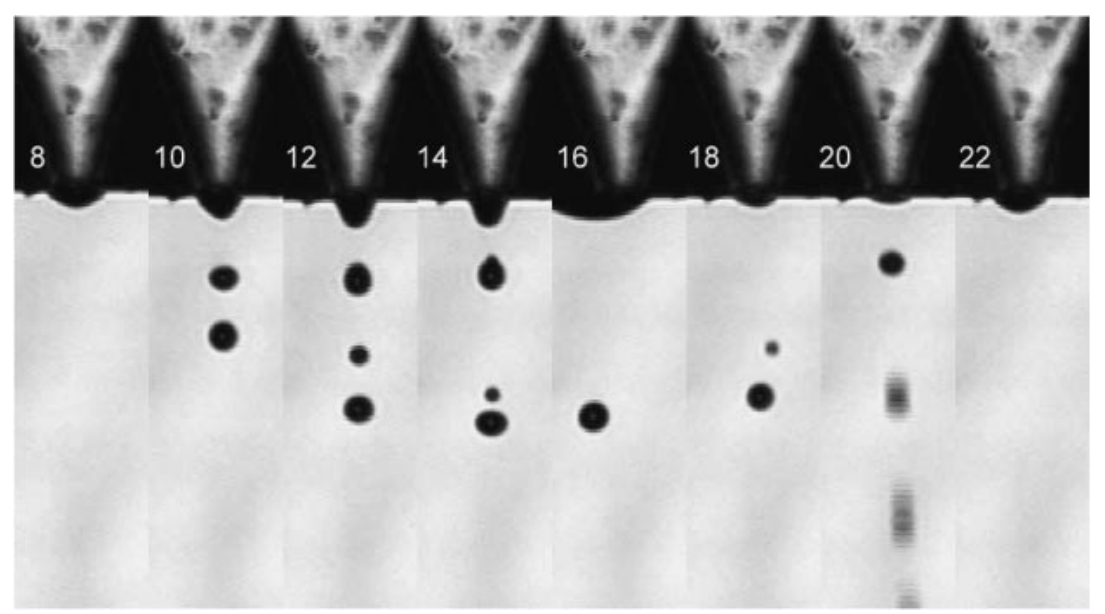

(a) $1 \_t_{d w e l l} 2 \_2 t_{d w e l l} \_1 \_10 \_1500$ with $t_{d w e l l}$ varying from $8 \mu \mathrm{s}$ to $22 \mu \mathrm{s}$.

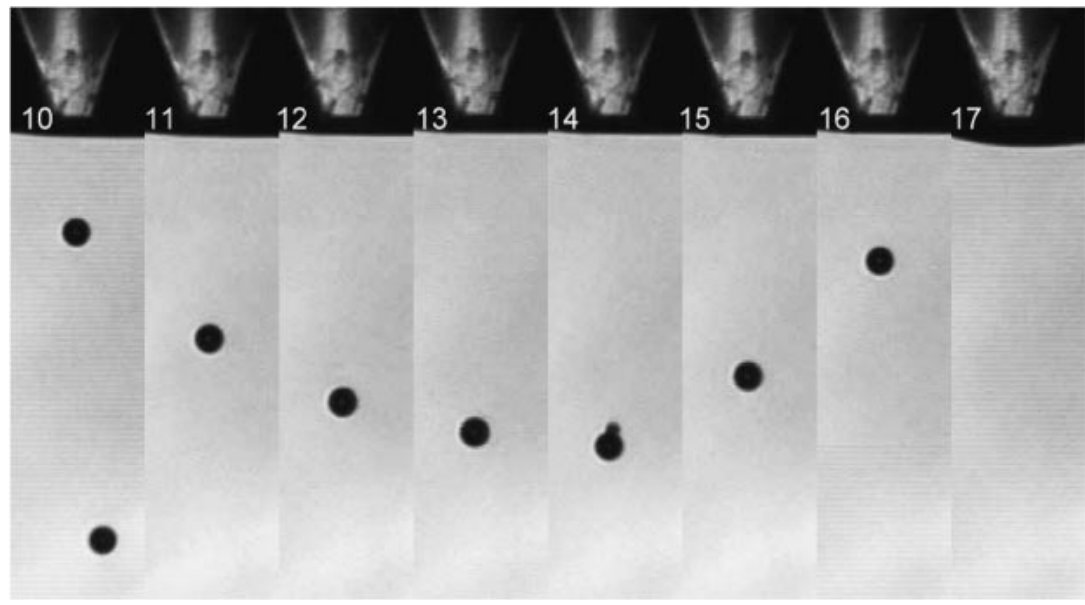

(b) $1 \_t_{d w e l l} \_2 \_2 t_{d w e l l} \_1 \_15 \_1500$ with $t_{d w e l l ~}$ varying from $10 \mu \mathrm{s}$ to $17 \mu \mathrm{s}$.

Fig. 8 Experimental observations of droplet with various $t_{\text {dewll }}$ for (a) DI water, (b) thylene glycol.

The decay time is approximately $500 \mu$ s for DI water under 1.5_15_3_30_1.5_8_Fr conditions and $44 \mu$ s for ethylene glycol under 1_13_2_26_1_15_Fr conditions. The decay time in ethylene glycol is shorter than that in DI water because the viscosity of ethylene glycol is substantially higher, resulting in greater damping. Thus, a higher pulse frequency can be used for ethylene glycol without the problems mentioned earlier.

\subsection{Pulse time effects}

Table 3 shows the experimental conditions used for investigation of the effects of positive voltage keeping time $\left(t_{\mathrm{dwell}}\right)$ on droplet ejection behavior for DI water and ethylene glycol. The results of droplet ejection are shown in Fig. 8, taken at $t=150 \mu \mathrm{s}$. Figure 8(a) shows the behaviors of DI water ejection under conditions of

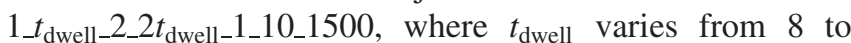
$22 \mu \mathrm{s}$. It is found that there is no droplet ejection for DI water when $t_{\text {dwell }}$ is below $10 \mu$ s or above $20 \mu \mathrm{s}$. Three droplets are observed when $t_{\mathrm{dwell}}$ equals to 10,12 , and $14 \mu \mathrm{s}$. Only one droplet is found in the picture if $t_{\text {dwell }}$ increases from 16 to $20 \mu \mathrm{s}$. The droplet morphologies for ethylene glycol under conditions of $1 \_t_{\text {dwell }} 2 \_2 t_{\text {dwell- } 1 \_15 \_1500,} t_{\text {dwell }}$ vary from 10

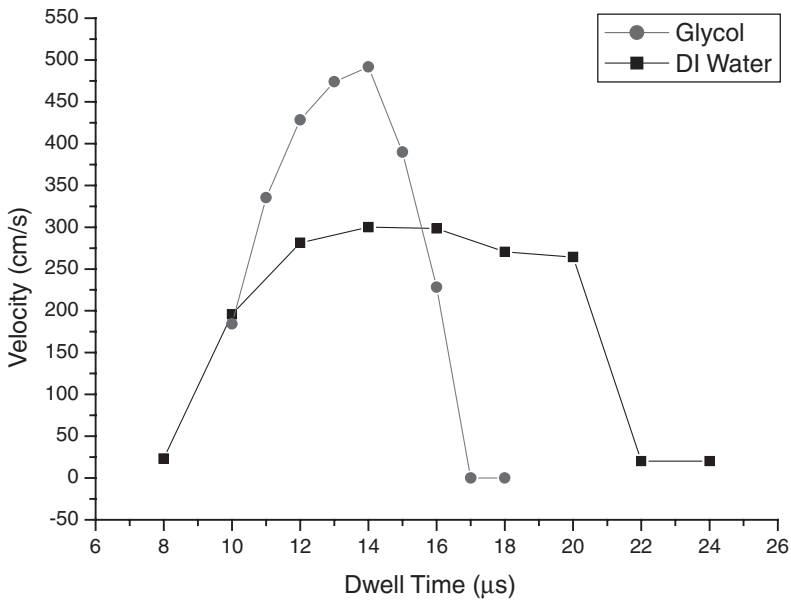

Fig. 9 Average droplet velocities at $150 \mu$ s for DI water and ethylene glycol under various dwell times.

to $17 \mu$ s are shown in Fig. 8(b). Two droplets are found in pictures when $t_{\text {dwell }}$ is $10 \mu \mathrm{s}$, and then reduces to one when $t_{\text {dwell }}$ increases. However, there is no droplet when $t_{\text {dwell }}=$ $17 \mu \mathrm{s}$. 


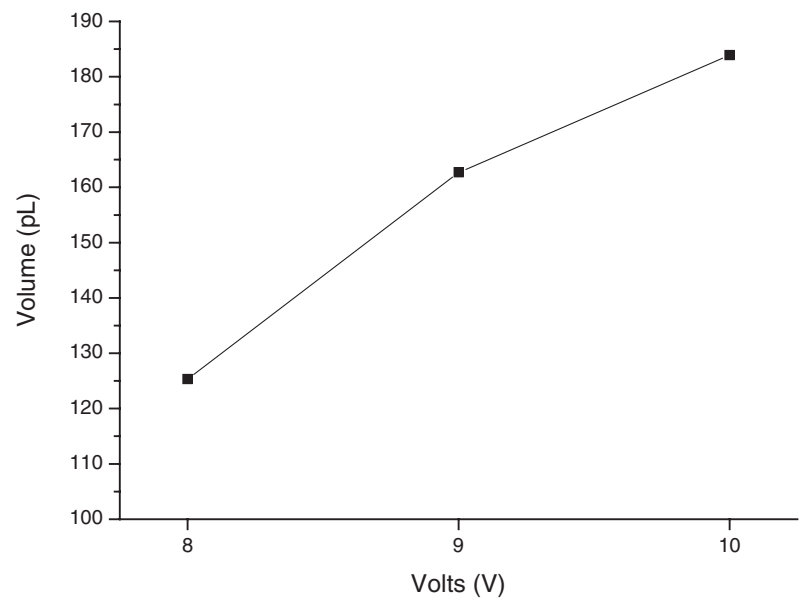

Fig. 10 Volume of main droplet for DI water at $200 \mu$ s under various voltages.

The relationships between average droplet velocity and positive voltage keeping time are shown in Fig. 9. The velocity variations with respect to $t_{\text {dwell }}$ for DI water and ethylene glycol follow the same trend from 0 to $150 \mu \mathrm{s}$. The droplet velocity increases with $t_{\text {dwell }}$ to a maximum value and then decreases as $t_{\text {dwell }}$ increases further. The maximum droplet velocity occurs at $t_{\text {dwell }}=14 \mu$ s for ethylene glycol. When $t_{\text {dwell }}$ is between $14-16 \mu \mathrm{s}$, the maximum droplet velocity will be achieved for DI water. As mentioned in Section 3.1, the optimal pulse time for DI water and ethylene glycol is 15.57 and $13.48 \mu$ s respectively. This is very consistence between theoretical calculation and experimental observation. If $t_{\text {dwell }}$ is selected randomly, the pressure wave distribution within the printhead is more chaotic, leading to decreased droplet ejection velocity and unsteady droplets.

\subsection{Voltage effects}

Table 4 shows the experimental conditions used for investigation of the effects of voltage magnitude on droplet ejection behavior for DI water and ethylene glycol. The voltages applied for DI water are 8,9 and 10 volts at conditions of 1_15_2_30_1_V_2000. Ethylene glycol is tested at 14,17 and 20 volts at conditions of $1 \_13 \_22_{-} 6 \_1 \_V \_2000$. As mentioned in Section 3.2, the droplet ejection behaviors for ethylene glycol are approximately identical when frequencies are below $22700 \mathrm{~Hz}$. Therefore, the same frequency of $2000 \mathrm{~Hz}$ was adopted for both of ethylene glycol and DI water.

Figure 10 shows the droplet volume variation with respect to voltage for DI water. All of diameters of droplets are taken from ejection images at $t=200 \mu \mathrm{s}$. The diameters are 62, 68 and $71 \mu \mathrm{m}$ respectively. Thus, the droplet volumes are 125 , 162 and $184 \mathrm{pL}$ respectively. An increase in the operating voltage results in a greater volume. Figure 11 shows the variations of droplet velocity with respect to time at different operating voltages for DI water. As the voltage increases, so does the droplet velocity. The maximum velocity in all cases occurs at $30 \mu \mathrm{s}$. From the theory of wave propagation as shown in Fig. 6, the maximum velocity of droplet at the nozzle should be occurred at time of $t=1.5 t_{\mathrm{opt}}$. It is equal to $23.4 \mu$ s for DI water. However, this value is less than the

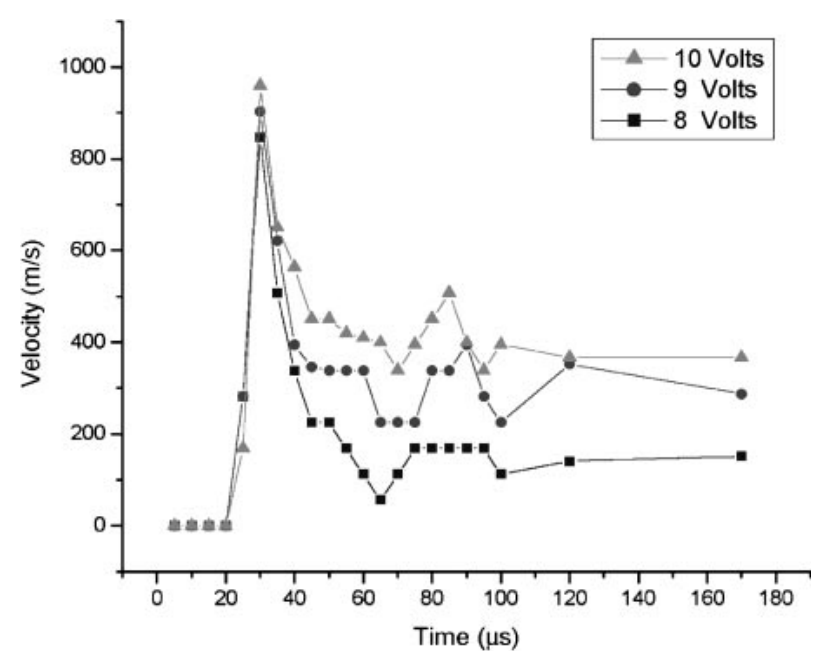

Fig. 11 Velocity of main droplet as functions of time for DI water under various voltages.

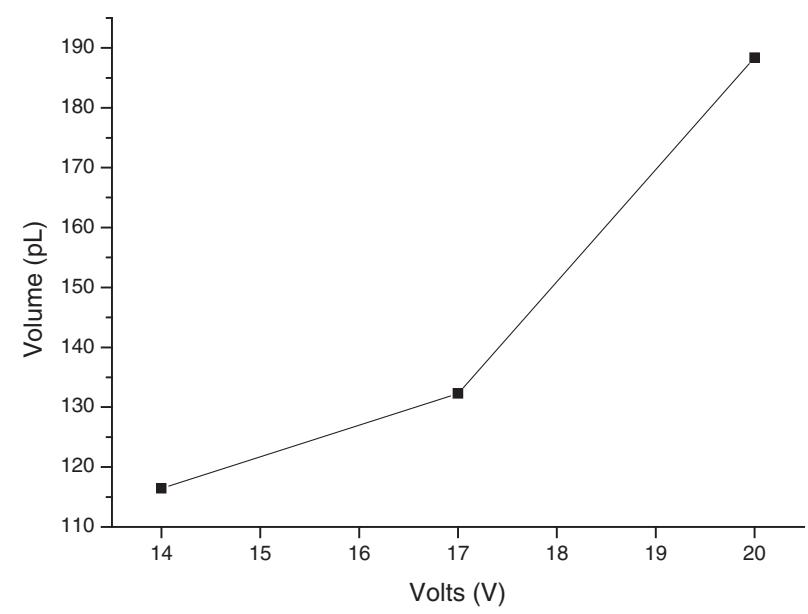

Fig. 12 Volume of main droplet for ethylene glycol at $200 \mu$ s under various voltages.

experimental results. Since the theoretical model for wave propagation is established under assumptions, such as that the time of $t_{\text {rise }}, t_{\text {fall }}$ and $t_{\text {finrise }}$ are ignored and the damping phenomena also does not consider. The droplet separates from the nozzle at nearly $65 \mu \mathrm{s}$, also longer than theoretical time $\left(=3.5 t_{\mathrm{opt}}=54.5 \mu \mathrm{s}\right)$. After that, the droplet is only affected by gravity.

Figure 12 shows the variations of droplet volumes under three different operating voltages of 14,17 and $20 \mathrm{~V}$ for ethylene glycol. All of the volumes are calculated from droplet diameters measured from ejection images at $t=$ $200 \mu \mathrm{s}$. The droplet diameters are 61, 63 and $71 \mu \mathrm{m}$ respectively, and corresponding volumes are 116, 132 and $188 \mathrm{pL}$. It is seen that the volume of droplet increases with operating voltage. Figure 13 shows the droplet velocity variations with respect to time at different operating voltages. The droplet velocity increases with voltage. However, all reaches a maximum value at about $30 \mu \mathrm{s}$. From the theoretical calculation, the maximum velocity of ethylene glycol droplet should occur at about $20.22 \mu$ s. Since the 


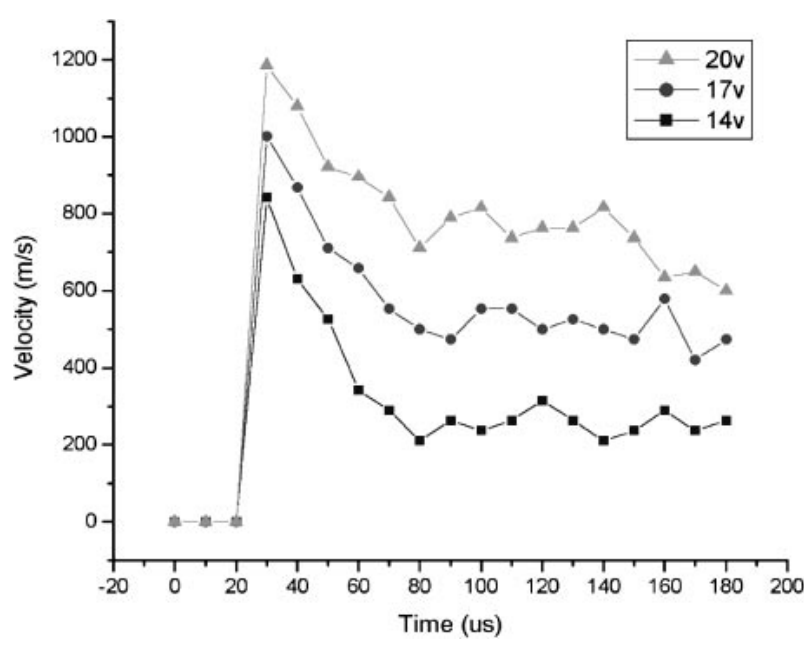

Fig. 13 Velocity of main droplet as function of time for ethylene glycol under various voltages.

viscosity of ethylene glycol is higher than DI water, damping effect is much than DI water is in the cavity. Hence, the experimental results of ethylene glycol deviates theoretical results more than DI water does. The droplet separates from the nozzle at nearly $t=90 \mu \mathrm{s}$. Afterward, the droplet velocity oscillates within a small range.

The selection of voltage magnitude should take into consideration the trade-offs between droplet velocity and volume. Increased voltage results in greater velocity, but also in larger droplet size, effectively reducing the print resolution. Increased resolution can be gained by using lower voltages at the expense of droplet velocity. The importance of these variables will depend greatly on the specific application.

\section{Summary}

In this study, the effects of operating frequency, positive voltage keeping time and voltage magnitude on droplet ejection behavior with double pulse voltage pattern for micro-droplet inkjet devices were investigated. Two types of liquid, DI water and ethylene glycol, are used in the experiments. The conclusions taken from this experiment are as follows.

(1) The droplet ejection behaviors can be successfully explained through the propagation theory of acoustic waves.

(2) The optimum operating frequencies for DI water and ethylene glycol are $2,000 \mathrm{~Hz}$ and $22,700 \mathrm{~Hz}$, respectively.

(3) When the positive voltage keeping time is equal to the time required for the pressure wave to propagate through the printhead, the velocity of the ejected droplet is maximized.

(4) As the voltage increases, the velocity and volume of the liquid droplet become larger.

\section{REFERENCES}

1) Q. Liu: J. Mater. Proc. Technol. 115 (2001) 271-283.

2) T. R. Hebner, C. C. Wu, D. Marcy, M. H. Lu and J. C. Strum: Appl. Phys. Lett. 72 (1998) 519-521.

3) K. Yamaguchi, K. Sakai, T. Yamanaka and T. Hirayama: Precision Eng. 24 (2000) 2-8.

4) L. P. Hue: J. Imaging Sci. Technol. 42 (1998) 49-62.

5) N. Bugdayci, D. B. Bogy and F. E. Talk: IBM J. Res. Dev. 27 (1983) $171-180$.

6) D. B. Bogy and F. E. Talk: IBM J. Res. Dev. 28 (1984) 314-321.

7) T. W. Shield, D. B. Bogy and F. E. Talke: IBM J. Res. Dev. 31 (1987) 96-110.

8) P. H. Chen, H. Y. Peng, H. Y. Liu, S. L. Chang, T. I. Wu and C. H. Cheng: Int. J. Mech. Sci. 41 (1999) 235-248. 